\title{
Longitudinal brain structural alterations and systemic inflammation in obstructive sleep apnea before and after surgical treatment
}

Wei-Che Lin ${ }^{1}$, Chih-Cheng Huang ${ }^{2}$, Hsiu-Ling Chen ${ }^{1,3}$, Kun-Hsien Chou ${ }^{4}$, Pei-Chin Chen ${ }^{1}$, Nai-Wen Tsai ${ }^{2}$, Meng-Hsiang Chen ${ }^{1}$, Michael Friedman ${ }^{5,6}$, Hsin-Ching Lin ${ }^{7,8^{*}+}$ and Cheng-Hsien Lu ${ }^{2,9^{*}+}$

\begin{abstract}
Background: Systemic inflammation, neurocognitive impairments, and morphologic brain changes are associated with obstructive sleep apnea (OSA). Understanding their longitudinal evolution and interactions after surgical treatment provides clues to the pathogenesis of cognitive impairment and its reversibility. In the present study, we investigate clinical disease severity, systemic inflammation, cognitive deficits, and corresponding gray matter volume (GMV) changes in OSA, and the modifications following surgery.
\end{abstract}

Methods: Twenty-one patients with OSA (apnea-hypopnea index, $\mathrm{AHI}>5$ ) and 15 healthy volunteers $(\mathrm{AHI}<5)$ underwent serial evaluation, including polysomnography, flow cytometry for leukocyte apoptosis categorization, cognitive function evaluation, and high-resolution brain scan. Disease severity, leukocyte apoptosis, cognitive function, and imaging data were collected to assess therapeutic efficacy 3 months after surgery.

Results: Pre-operatively, patients presented with worse cognitive function, worse polysomnography scores, and higher early leukocyte apoptosis associated with increased insular GMV. There was reduced GMV in the anterior cingulate gyrus before and after surgery in the cases compared to that in controls, suggesting an irreversible structural deficit. Post-operatively, there were significant improvements in different cognitive domains, including attention, executive and visuospatial function, and depression, and in early leukocyte apoptosis. There was also a significant decrease in GMVs after treatment, suggesting recovery from vasogenic edema in the precuneus, insula, and cerebellum. Improvement in early leukocyte apoptosis post-surgery predicted better recovery of precuneus GMV.

Conclusions: In OSA, increased disease severity and systemic inflammation can alter GMV in vulnerable regions. Surgical treatment may improve disease severity and systemic inflammation, with subsequent recovery in brain structures and functions.

Keywords: Obstructive sleep apnea, Voxel-based morphometry, Magnetic resonance imaging, Oxidative stress, Leukocyte apoptosis

\footnotetext{
*Correspondence: hclin@cgmh.org.tw; chlu99@ms44.url.com; chlu99@cgmh.org.tw

${ }^{\dagger}$ Cheng-Hsien Lu and Hsin-Ching Lin contributed equally to this work ${ }^{2}$ Department of Neurology, Kaohsiung Chang Gung Memorial Hospital,

Chang Gung University College of Medicine, Kaohsiung, Taiwan

${ }^{7}$ Department of Otolaryngology, Kaohsiung Chang Gung Memorial Hospital, Chang Gung University College of Medicine, 123, Ta Pei Road, Niao Sung District, Kaohsiung, Taiwan

Full list of author information is available at the end of the article
} 


\section{Background}

Obstructive sleep apnea (OSA) is characterized by repetitive episodes of apnea or hypopnea during sleep. Sleep fragmentation and intermittent hypoxia in OSA can trigger elevated oxidative stress and subsequent inflammation [1]. Several imaging studies have demonstrated structural and functional changes in the brains of patients with OSA, with corresponding neuropsychological impairments [2]. Treatments, such as continuous positive airway pressure (CPAP), can significantly improve memory, attention, and executive function that parallels correction of the structural deficits. These data can shed light for treatment approaches for OSA and serve as motivators for treatment adherence.

The role of peripheral blood inflammatory markers related to OSA has not yet been systematically investigated. Several systemic inflammatory markers are involved in endothelial damage that occurs during repetitive hypoxia/re-oxygenation injury [3]. The interface between the vascular endothelium and host inflammatory response may also be related to the activation of resident microglia and peripheral blood leukocytes [4], as well as the expression of inflammatory cytokines and adhesion molecules [5]. However, the role of these inflammatory markers in the pathogenesis of OSA, especially in its relationship with structural brain alterations and cognitive function, as well as the effects of different treatments on these markers, remains unclear.

Previous studies have demonstrated that direct oxidative damage associated with sleep deprivation occurs across many brain regions [6], and chronic intermittent hypoxia can increase brain cortical neuronal cell death [7]. Structural evaluation, such as voxel-based morphometry (VBM), is an assumption-free, objective, and operator-independent method that applies voxel-wise comparisons throughout the brain to detect differences in gray matter volume (GMV). Extensive morphological alterations in several brain areas in patient with OSA had previously been shown by VBM [8]. However, the reported structural changes in OSA have been highly variable due to different VBM pre-processing pipelines and statistical threshold settings used in studies. The therapeutic effect of CPAP on structural brain alterations is still not conclusive [9-11]. CPAP compliance varies from 28 to $80 \%[12,13]$ and there is a consensus that a certain number of OSA patients cannot or will not use CPAP. Surgery for OSA is not a substitute for CPAP, but a salvage procedure for those who have failed CPAP and other conservative therapies. No research to date has focused on the effects of surgery in OSA using the relatively optimized longitudinal VBM method.

Serial evaluation before and after surgical treatment, and comparison with data from healthy controls, may elucidate the underlying morphologic, inflammatory, cognitive, and clinical performance changes in OSA patients and is essential to improving therapeutic strategies. This study aimed to determine (i) if OSA patients demonstrate alteration of GMV after surgical treatment, (ii) if changes in GMV are affected by the baseline or changes of systemic inflammation and their relationship to disease severity after surgery; and (iii) if there is any correlation between GMV and cognitive performance before and after treatment.

\section{Methods \\ Participants}

This prospective study targeted patients with OSA [apnea-hypopnea index $(\mathrm{AHI}) \geq 5$ ] evaluated for surgical treatment. Twenty-one patients (18 men and 3 women; mean age, $40.14 \pm 10.80$ years; body mass index [BMI], $26.24 \pm 3.40 \mathrm{~kg} / \mathrm{m}^{2}$; AHI, $38.77 \pm 19.91$ events $\left./ \mathrm{hr}\right)$ and 15 healthy volunteers $(\mathrm{AHI}<5)(11$ men and 4 women; mean age, $39.80 \pm 9.53$ years; BMI, $23.97 \pm 2.50 \mathrm{~kg} / \mathrm{m}^{2}$, AHI, $2.43 \pm 1.61$ events/hr) were recruited. The Hospital Ethics Committee approved the study and all of the participants provided written informed consent.

All of the control subjects and OSA patients, who had failed CPAP and other conservative therapies, were enrolled through the sleep center after presenting with snoring. All participants underwent similar evaluations, including clinical characteristics, overnight polysomnography (PSG), blood samples for assessment of leukocyte apoptosis, neuropsychological tests (NP), and magnetic resonance imaging (MRI) for brain structure calculation. The last three evaluations were done on the same day and within 2 weeks of PSG.

Overnight PSG was assessed according to a previous report [14]. All-night comprehensive diagnostic sleep studies were performed at the hospital's sleep center. The severity of sleep-disordered breathing was classified according to the number of apneas and hypopneas during sleep, and was classified as normal for AHI 0-5, mild for AHI 5-15, moderate for AHI 15-30, and severe for AHI $>30$ [15]. Central respiratory events were excluded from the severity classification. All PSGs were scored and read by a board-certified physician blinded to the study. Patients were excluded if they had a history of major mental disorder, brain injury or illness, diabetes mellitus, cerebrovascular disease, major cardiovascular disorder (e.g., stroke, heart failure, myocardial infarction), or central/peripheral disorders known to affect the autonomic nervous systems.

Concepts of surgical treatment for OSA are based on reducing the volume of redundant tissues, stiffening the flaccid soft palate, and suspending the collapsed tongue base to maintain airway patency in order to improve 
symptoms and reduce the sequelae of OSA. The efficacy and safety of multi-level surgery has been previously demonstrated [16]. All of the surgical procedures were performed by the co-corresponding author ( $\mathrm{H}-\mathrm{C}$ Lin) under general anesthesia, with orotracheal intubation. The techniques used were determined at the discretion of the treating sleep surgeon based on the severity of OSA by PSG, and upper airway abnormalities were examined by flexible fibroscopy. The surgical techniques were as previously described [17-19].

\section{Assessment of leukocyte apoptosis}

Leukocyte apoptosis, and its subtypes, were identified according to a previous study [20] with APO 2.7-phycoerythrin (PE), early apoptosis, and late apoptosis. Positive APO 2.7-PE indicated apoptotic cells. Further analysis of early and late apoptosis was conducted by annexin V-FITC and 7-aminoactinomycin D (7-AAD). Late apoptotic cells demonstrated disruption of the cell membrane integrity while early apoptotic cells demonstrated early and reversible apoptotic changes. Results were expressed as percentages of specific fluorescence-positive cells.

\section{Neuropsychological tests}

The NP battery of tests focused on attention, execution function, speech and language, and mnemonic and visuoconstruction abilities. Different domain evaluations were measured as in previous studies [21, 22] with subtests from the Cognitive Ability Screening Instrument (CASI) [23] and the Wechsler Adult Intelligence Scale (WAIS-III) [24]. The Beck Depression Inventory II (BDI), was used to evaluate severity of depression [25].

\section{MRI image acquisition}

All cross-sectional and longitudinal volumetric structural MRI was performed on identical 3-Tesla GE Signa wholebody MRI systems (General Electric Healthcare, Milwaukee, WI, USA) equipped with an eight-channel head coil at the Kaohsiung Chang Gung Memorial Hospital in Taiwan. A T1-weighted three dimensional fluid-attenuated inversion-recovery fast spoiled gradient-recalled echo pulse sequence was used with following imaging parameters for each participant and time-point: TR/TE/ $\mathrm{TI}=9.5 / 3.9 / 450 \mathrm{~ms}$; flip angle, $15^{\circ} ; \mathrm{NEX}=1$; matrix size $=512 \times 512$; voxel size $=0.47 \times 0.47 \times 1.3 \mathrm{~mm}^{3}$; and 110 axial slices.

An experienced neuroradiologist, blinded to the participants' status, visually examined all of the MR scans. None of the participants in the study were excluded.

\section{Imaging data analysis}

Cross-sectional VBM processing To identify regional GMV differences between patients with OSA, before and after surgical treatment, and the healthy control group, structural T1-weighted images were processed using statistical parametric mapping (SPM8; http://www.fil.ion. ucl.ac.uk/spm; Wellcome Institute of Neurology, University College London, UK) and the VBM8 toolbox (http:// dbm.neuro.uni-jena.de) with default settings as described in the manual. The procedure for the cross-sectional VBM pipeline followed that of previous cross-sectional based VBM studies from our group [26, 27] (additional details available in the Method of the online "Additional file 1").

Longitudinal VBM processing The default longitudinal batch script in the VBM8 toolbox was used to identify longitudinal effects to GMV in patients with OSA before and after surgical treatment. In this pipeline the modulation step was not used because our focus was on relative tissue differences between different time-points within the same participant [27]. First, follow-up (after surgery) T1-weighted scan was registered to the baseline scan (before surgery). Second, the mean anatomic image was calculated using the realigned images and served as a reference image for realignment of baseline and follow-up scans for each participant. Third, the individual realigned baseline and follow-up scans were bias corrected to account for field inhomogeneities regarding the corresponding mean anatomical scan. Fourth, the resultant biascorrected mean anatomical scan and realigned images were segmented into GM, WM, and CSF tissue segments using the VBM8 segmentation approach. Fifth, the DARTEL registration parameters were estimated using the GM tissue segments of the bias-corrected mean anatomical scan. The resulting registration parameters were applied to the corresponding tissue segments of the realigned baseline and follow-up anatomical scans. The resulting normalized GM segments for each time point for each participant were smoothed using an 8-mm FWHM Gaussian kernel and served as inputs for the subsequent longitudinal statistical model.

\section{Statistical analysis \\ Analysis of demographic data between groups}

All statistical analyses of demographic data, including clinical profiles, laboratory data, global tissue volume, and NP tests, were performed using the independent $t$ test, Pearson's Chi square test, and analysis of covariance (ANCOVA), as appropriate (details of statistical analyses are noted in the legend of Table 1). The longitudinal data were compared in the patient group, before and after surgical treatment, using a paired t-test. All statistical significance was set at $p<0.05$ (version 12, SPSS Inc., Chicago, IL, USA). 
Table 1 Demographic characteristics of OSA patients and control subjects

\begin{tabular}{|c|c|c|c|c|c|c|}
\hline & NC (controls) & OSA $_{\text {baseline }}$ & OSA $_{\text {follow }}$ & $p$ value & & \\
\hline & & & & $\begin{array}{l}\text { NC vs. OSA- } \\
\text { baseline }\end{array}$ & $\begin{array}{l}\text { NC vs. } \\
\text { OSA }_{\text {follow }}\end{array}$ & $\begin{array}{l}\text { OSA }_{\text {baseline }} \text { vs. } \\
\text { OSA follow }\end{array}$ \\
\hline Number & 15 & 21 & & & & \\
\hline $\operatorname{Sex}(M: F)$ & $11: 4$ & $18: 3$ & & 0.418 & & \\
\hline Age (years) & $39.80(9.53)$ & $40.14(10.80)$ & & 0.922 & & \\
\hline BMI $\left(\mathrm{kg} / \mathrm{m}^{2}\right)$ & $23.97(2.50)$ & $26.24(3.40)$ & $25.83(3.73)$ & 0.050 & 0.130 & $0.033^{*}$ \\
\hline HbA1c (\%) & $5.54(0.25)$ & $5.80(0.46)$ & $5.75(0.54)$ & 0.206 & 0.338 & 0.537 \\
\hline Sugar (mg/dl) & $92.00(8.04)$ & $93.43(12.14)$ & $95.05(12.40)$ & 0.925 & 0.546 & 0.217 \\
\hline hs-CRP (mg/dl) & $0.80(0.81)$ & $3.22(4.30)$ & $2.32(2.62)$ & 0.108 & 0.069 & 0.292 \\
\hline Total cholesterol & $192.10(34.93)$ & $191.96(29.98)$ & $194.57(32.00)$ & 0.880 & 0.985 & 0.599 \\
\hline Triglyceride & $120.87(95.78)$ & $156.13(107.13)$ & $154.87(79.05)$ & 0.441 & 0.343 & 0.926 \\
\hline $\mathrm{HDL}$ & $62.67(14.44)$ & $54.70(10.57)$ & $53.39(10.32)$ & 0.085 & 0.056 & 0.393 \\
\hline LDL & $106.40(34.41)$ & $122.04(29.46)$ & $115.04(39.67)$ & 0.171 & 0.540 & 0.318 \\
\hline Smoking (yes/no) & $(14 / 1)$ & $(18 / 3)$ & $(19 / 2)$ & 0.728 & 1.000 & 1.000 \\
\hline Voxel-based morphometry & & & & & & \\
\hline $\mathrm{GMV}(\mathrm{ml})$ & $613.32(49.33)$ & $620.69(72.14)$ & $620.86(72.69)$ & 0.707 & 0.702 & 0.953 \\
\hline WMV (ml) & $587.49(59.16)$ & $583.96(53.91)$ & $582.17(50.92)$ & 0.851 & 0.722 & 0.373 \\
\hline $\operatorname{CSFV}(\mathrm{ml})$ & $216.52(25.02)$ & $215.96(22.39)$ & $216.81(22.52)$ & 0.943 & 0.971 & 0.477 \\
\hline $\operatorname{TIV}(\mathrm{ml})$ & $1417.33(113.22)$ & $1420.61(119.15)$ & $1419.83(119.06)$ & 0.931 & 0.947 & 0.674 \\
\hline Polysomnography (PSG) & & & & & & \\
\hline $\mathrm{AHI}$ & $2.43(1.61)$ & $38.77(19.91)$ & $25.21(20.93)$ & $0.000^{*}$ & $0.000^{*}$ & $0.004^{*}$ \\
\hline De-saturation index & $0.77(0.82)$ & $26.59(19.38)$ & $16.88(19.23)$ & $0.000^{*}$ & $0.006^{*}$ & $0.009^{*}$ \\
\hline Average $\mathrm{O}_{2}$ saturation & $97.09(0.82)$ & $95.59(1.39)$ & $95.81(1.79)$ & $0.004^{*}$ & 0.053 & 0.534 \\
\hline Snoring index & $208.96(240.67)$ & $385.71(179.17)$ & $323.52(181.58)$ & 0.059 & 0.278 & 0.116 \\
\hline Systolic BP (mmHg) & $124.90(15.86)$ & $135.58(15.38)$ & $132.07(11.58)$ & 0.538 & 0.497 & 0.323 \\
\hline Diastolic BP (mmHg) & $75.53(9.09)$ & $84.58(11.00)$ & $82.55(12.01)$ & 0.082 & 0.142 & 0.469 \\
\hline Leukocyte apoptosis (\%) & & & & & & \\
\hline Granulocyte APO 2.7 apoptosis & $0.429(0.219)$ & $0.894(1.012)$ & $0.831(0.940)$ & 0.160 & 0.125 & 0.830 \\
\hline Monocyte APO 2.7 apoptosis & $2.135(1.410)$ & $3.156(3.321)$ & $2.570(2.903)$ & 0.439 & 0.575 & 0.585 \\
\hline Lymphocyte APO 2.7 apoptosis & $0.481(0.226)$ & $0.658(0.444)$ & $0.475(0.255)$ & 0.283 & 0.835 & 0.164 \\
\hline Total leukocyte APO 2.7 apoptosis & $0.891(0.426)$ & $1.442(1.084)$ & $1.271(1.004)$ & 0.128 & 0.236 & 0.626 \\
\hline Granulocyte late apoptosis & $11.726(6.986)$ & $16.667(10.032)$ & 14.599 (14.039) & 0.107 & 0.331 & 0.622 \\
\hline Granulocyte early apoptosis & $13.920(5.615))$ & $23.117(11.007)$ & $18.250(12.008)$ & $0.010^{*}$ & 0.302 & 0.099 \\
\hline Monocyte late apoptosis & $12.593(7.401)$ & $12.480(8.384)$ & $14.520(8.876)$ & 0.936 & 0.428 & 0.318 \\
\hline Monocyte early apoptosis & $15.263(5.068)$ & $23.254(8.033)$ & $16.796(8.342)$ & $0.002^{*}$ & 0.579 & $0.007^{*}$ \\
\hline Lymphocyte late apoptosis & $2.331(1.090)$ & $2.306(0.915)$ & $2.577(1.178)$ & 0.906 & 0.647 & 0.601 \\
\hline Lymphocyte early apoptosis & $5.410(1.988)$ & $6.716(2.505)$ & $6.600(2.624)$ & 0.147 & 0.210 & 0.981 \\
\hline Total leukocyte late apoptosis & $6.875(2.588)$ & $7.541(3.170)$ & $7.972(4.126)$ & 0.407 & 0.174 & 0.744 \\
\hline Total leukocyte early apoptosis & $9.203(1.987)$ & $13.535(3.713)$ & $10.599(4.431)$ & $0.000^{*}$ & 0.286 & $0.021^{*}$ \\
\hline
\end{tabular}

Data are presented as mean (SD)

Age data was compared by independent t-test; sex data was compared by Pearson's Chi square test; BMI data was compared by ANCOVA after controlling for age and sex; Sugar, HbA1c, hs-CRP, smoking, voxel-based morphometry measurement, polysomnography result and leukocyte apoptosis data were compared by ANCOVA after controlling for age, sex, and BMI

AHI apnea-hypopnea index; ANCOVA Analysis of Covariance; BMI, body mass index, CSFV cerebrospinal fluid volume; GMV gray matter volume; HDL high-density lipoprotein cholesterol; $h s$-CRP high sensitivity C-reactive protein; LDL low-density lipoprotein cholesterol; NC normal controls; OSA obstructive sleep apnea; TIV total intracranial volume; $W M V$ white matter volume

NC vs. OSA baseline: $_{\text {Statistical comparison was performed by ANCOVA between controls and OSA }}$ baseline

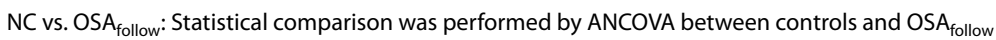

OSA $_{\text {baseline }}$ Vs. OSA $A_{\text {follow: }}$ : Statistical comparison was performed by paired t test between OSA $\mathrm{baseline}_{\text {and OSA }}$ follow

* Statistical threshold was set at $p<0.05$ 


\section{Group comparisons of GMV}

Cross-sectional (patient with OSA before/after surgical treatment vs. healthy control) and longitudinal (patient with OSA before and after surgical treatment) voxel-wise GMV differences were examined using ANCOVA (with age, sex, and BMI as covariates) and paired t-test with SPM8, respectively. To prevent possible partial volume effects around the border between different tissue segments, voxels with an absolute GM probability $<0.2$ were excluded in the statistical analysis.

Whole-brain statistical inferences were considered significant at family-wise error (FWE) corrected $p<0.05$ using the cluster-extend threshold approach with 3dClusterSim in AFNI (Analysis of Functional NeuroImages, http://afni.nimh.nih.gov/afni/). Based on the results of the Monte Carlo simulation, a cluster size of at least 453 voxels and at least 639 voxels were considered statistically significant for the cross-sectional and longitudinal designs, respectively [3dClusterSim using the following parameters: single voxel $p<0.01$, FWHM $=8 \mathrm{~mm}$ (cross-sectional)/9 mm (longitudinal) with GM mask and 10,000 simulations].

\section{Correlation among GMV, clinical profile, leukocyte apoptosis, and NP tests}

The mean GMV was extracted from the clusters, with significant statistical difference from the group comparisons, for further correlation analysis. Partial Pearson correlation analysis adjusted for age, gender, and BMI was performed to correlate GMVs showing significant differences in cross-sectional and longitudinal effect with clinical severity, leukocyte apoptosis, and NP tests. Significance was set at a Bonferroni corrected $p<0.05$, accounting for multiple ROI comparisons.

\section{Results}

\section{Demographic characteristics of the participants}

Based on the demographic characteristics of the 21 OSA cases and 15 controls (Table 1), there were no significant differences in age, sex, or BMI between the OSA baseline $\left(\mathrm{OSA}_{\text {baseline, }}\right.$ before surgical treatment) and

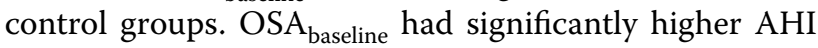
$(p<0.001)$, higher desaturation index $(p<0.001)$, and lower average $\mathrm{O}_{2}$ saturation $(p=0.004)$ during sleep, compared with controls. After surgery, the OSA followup group $\left(\mathrm{OSA}_{\text {follow }}\right)$ still had significantly higher AHI $(p<0.001)$ and desaturation index $(p=0.006)$ compared with controls.

After surgical treatment, $\mathrm{OSA}_{\text {follow }}$ had significant improvements in BMI $(p=0.033)$, AHI $(p=0.004)$, and desaturation index $(p=0.009)$ compared with $\mathrm{OSA}_{\text {baseline. }}$

\section{Group comparisons of leukocyte apoptosis}

Percentages of early apoptosis of total leukocytes $(p<0.001)$, granulocytes $(p=0.010)$, and monocytes $(p=0.002)$ were significantly higher in OSA $_{\text {baseline }}$ than in the controls (Table 1).

In contrast, there were no significant differences in the percentages of any kind of leukocyte apoptosis between $\mathrm{OSA}_{\text {follow }}$ and controls.

Nonetheless, $\mathrm{OSA}_{\text {follow }}$ showed significantly decreased percentages of early apoptosis of total leukocytes $(p=0.021)$ and monocytes $(p=0.007)$ compared with OSA $_{\text {baseline. }}$

\section{Group comparisons of NP tests}

$\mathrm{OSA}_{\text {baseline }}$ had worse short term memory scores compared with controls $(p=0.048)$ (Table 2). OSA $_{\text {follow }}$ also had improvements in BDI compared with controls $(p=0.048)$.

$\mathrm{OSA}_{\text {follow }}$ showed significant improvements in attention (processing speed, $p<0.001$ ), executive function (digit symbol, $p=0.001$ ), visuospatial function (block design, $p=0.015$ ), and depression (BDI, $p=0.011$ ).

\section{Group comparisons of gray matter volume (GMV)}

Regions of GMV difference between pre- and post-operative scans of patients and controls, and the longitudinal changes before and after surgery in the OSA group are presented in Table 3 and Fig. 1.

$\mathrm{OSA}_{\text {baseline }}$ showed significantly lower GMV in the left anterior cingulate gyrus and higher GMV in the right insula compared with controls. OSA $_{\text {follow }}$ showed significantly lower GMV in the left anterior cingulate gyrus but not significantly higher GMV in the right insula when compared with controls.

An exploratory group-wise comparison of the OSA group before and after surgical treatment revealed that after surgery, patients exhibited decreased GMV in the left hippocampus/posterior cingulate gyrus/precuneus (Hipp/PCG/Precuneus) and left superior temporal gyrus/inferior frontal gyrus/insula (STG/IFG/Insula), and increased GMV in the left cerebellum (Fig. 2).

\section{Correlations between extracted regional GMV and all assessments Correlations with clinical profiles}

Before surgical treatment, worse PSG parameters (including higher $\mathrm{AHI}$ and desaturation index, and lower average $\mathrm{O}_{2}$ saturation) were associated with higher GMV in the right insula $(p / r=0.003 / 0.516 ; 0.003 / 0.508$; $0.001 /-0.574)$. After surgical treatment, there was no statistically significant correlation between PSG parameters and regional GMV (Table 4). 
Table 2 Cognitive test results of OSA patients and control subjects

\begin{tabular}{|c|c|c|c|c|c|c|}
\hline \multirow[t]{2}{*}{ Neuropsychological tests } & \multirow[t]{2}{*}{$N C(n=15)$} & \multirow{2}{*}{$\begin{array}{l}\text { OSA }_{\text {baseline }} \\
(n=21)\end{array}$} & \multirow{2}{*}{$\begin{array}{l}O_{\text {OSA }} \text { follow } \\
(n=21)\end{array}$} & \multicolumn{3}{|l|}{$p$ value } \\
\hline & & & & NC vs. OSA $A_{\text {baseline }}$ & NC vs. OSA follow & OSA $_{\text {baseline }}$ vs. OSA follow \\
\hline \multicolumn{7}{|l|}{ Attention } \\
\hline Mental control (CASI) & $9.53(0.99)$ & $9.40(1.70)$ & $9.50(1.10)$ & 0.664 & 0.908 & 0.776 \\
\hline Attention (CASI) & $7.73(0.59)$ & $7.65(0.81)$ & $7.60(0.68)$ & 0.999 & 0.631 & 0.716 \\
\hline Orientation (CASI) & $17.93(0.26)$ & $17.50(1.82)$ & $17.85(0.49)$ & 0.366 & 0.908 & 0.427 \\
\hline Processing speed (WAIS-III) & $105.67(18.36)$ & $100.55(9.24)$ & $108.55(11.81)$ & 0.280 & 0.757 & $<0.001^{*}$ \\
\hline \multicolumn{7}{|l|}{ Executive function } \\
\hline Digit symbol (WAIS-III) & $10.73(3.04)$ & $10.15(1.81)$ & $11.50(2.50)$ & 0.458 & 0.563 & $0.001^{*}$ \\
\hline Abstraction (CASI) & $9.73(2.05)$ & $10.25(1.41)$ & $10.70(1.17)$ & 0.645 & 0.145 & 0.119 \\
\hline \multicolumn{7}{|l|}{ Memory function } \\
\hline Long-term memory (CASI) & $10.00(0.00)$ & $10.00(0.00)$ & $10.00(0.00)$ & 1.000 & 1.000 & 1.000 \\
\hline Short-term memory (CASI) & $11.01(1.01)$ & $10.06(1.56)$ & $10.67(1.50)$ & $0.048^{*}$ & 0.398 & 0.175 \\
\hline \multicolumn{7}{|c|}{ Speech and language function } \\
\hline Language (CASI) & $9.78(0.46)$ & $9.95(0.22)$ & $9.95(0.22)$ & 0.218 & 0.320 & 1.000 \\
\hline Verbal fluency (CASI) & $9.27(1.03)$ & $8.95(1.57)$ & $9.30(1.08)$ & 0.265 & 0.550 & 0.330 \\
\hline \multicolumn{7}{|l|}{ Visuo-spatial function } \\
\hline Picture completion (WAIS-III) & $11.20(2.04)$ & $11.10(2.15)$ & $11.55(2.70)$ & 0.495 & 0.911 & 0.206 \\
\hline $\begin{array}{l}\text { Letter number search (WAIS- } \\
\text { III) }\end{array}$ & $9.53(3.27)$ & $9.45(2.09)$ & $9.60(3.27)$ & 0.791 & 0.959 & 0.780 \\
\hline Block design (WAIS-III) & $11.47(3.36)$ & $11.10(2.43)$ & $11.95(2.91)$ & 0.455 & 0.846 & $0.015^{*}$ \\
\hline Drawing (CASI) & $9.87(0.52)$ & $9.95(0.22)$ & $9.90(0.31)$ & 0.693 & 0.901 & 0.577 \\
\hline CASI total score & $94.85(3.70)$ & $94.01(4.78)$ & $95.47(3.39)$ & 0.357 & 0.809 & 0.163 \\
\hline BDI & $8.47(7.58)$ & $8.50(8.16)$ & $4.70(6.11)$ & 0.675 & $0.048^{*}$ & $0.011^{*}$ \\
\hline
\end{tabular}

Data are presented as mean (SD)

All data were compared by ANCOVA after controlling for age, sex, and BMI

AHI apnea-hypopnea index; ANCOVA Analysis of Covariance; BDI Beck Depression Inventory; BMI body mass index, CASI Cognitive Ability Screening Instrument; NC normal controls; OSA obstructive sleep apnea; WAIS Wechsler Adult Intelligence Scale

NC vs. OSA baseline: $_{\text {Statistical comparison was performed by ANCOVA between controls and OSA }}$ baseline

$\mathrm{NC}$ vs. OSA $\mathrm{follow}_{\text {: }}$ Statistical comparison was performed by ANCOVA between controls and OSA $\mathrm{A}_{\text {follow }}$

OSA $_{\text {baseline }}$ vs. OSA $\mathrm{follow}_{\text {: Statistical comparison was performed by paired t test between OSA }}$ baseline and OSA $\mathrm{A}_{\text {follow }}$

* Statistical threshold was set at $p<0.05$

Improvements in PSG parameters (follow-baseline) revealed no statistically significant correlation with regional GMV changes (follow-baseline).

\section{Correlations with leukocyte apoptosis}

Before surgical treatment, higher percentages of early apoptosis in granulocytes, monocytes, and total leukocytes were associated with higher GMV in the right insula $(p / r=0.017 / 0.419 ; 0.041 / 0.364 ; 0.022 / 0.405)$ (Table 4).

Improvements (follow-baseline) in early apoptosis of granulocytes and total leukocytes were significantly associated with recovery of GMV (follow-baseline) in the left Hipp/PCG/Precuneus $(p / r=0.022 / 0.521$; 0.012/0.562) (Table 4; Fig. 3). Correlation between improvements (follow-baseline) in the early apoptosis of monocytes and total leukocytes, and recovery of GMV (follow-baseline) in the cerebellum tended to significance $(p / r=0.060 /-0.438 ; 0.058 /-0.442)$.

\section{Correlations with NP tests}

There was no statistically significant correlation between differences in regional GMV and NP tests.

After surgical treatment, OSA patients demonstrated improvements in cognitive function. However, there was no statistically significant correlation between changes in regional GMV (follow-baseline) and NP tests (follow-baseline).

\section{Discussion}

\section{Summary}

(1) Longitudinal evaluation showed that surgery significantly improved AHI score, desaturation index, cognitive function, and decreased early leukocyte apoptosis.

(2) OSA patients may undergo different types of GMV changes before and after surgery.

(3) Before treatment, increased GMV in the insula was associated with increased disease sever- 
Table 3 Regions of GMV difference between pre- and post-operative scans of the patients and controls, and preand post-operative longitudinal changes in the OSA group

\begin{tabular}{|c|c|c|c|c|c|c|}
\hline \multicolumn{3}{|c|}{ MNI atlas coordinates } & \multirow[t]{2}{*}{ Cluster size } & \multirow[t]{2}{*}{ Anatomical region } & \multirow[t]{2}{*}{ Brodmann area } & \multirow[t]{2}{*}{ t-score } \\
\hline$x$ & $\mathrm{Y}$ & $\mathbf{z}$ & & & & \\
\hline \multicolumn{7}{|c|}{ Regional GMV decrease in OSA baseline $_{\text {Vs. NC }}$} \\
\hline-4 & 28 & 19 & 456 & Left Anterior Cingulate Gyrus & 24 & 3.41 \\
\hline \multicolumn{7}{|c|}{ Regional GMV increase in OSA baseline Vs. NC } \\
\hline 40 & -7 & -6 & 612 & Right Insula & 13 & 2.99 \\
\hline \multicolumn{7}{|c|}{ Regional GMV decrease in OSA follow $\mathrm{Vs}$. NC } \\
\hline-4 & 28 & 19 & 663 & Left Anterior Cingulate Gyrus & 24 & 3.65 \\
\hline \multicolumn{7}{|c|}{ Regional GMV decrease in OSA follow vs. OSA $A_{\text {baseline }}$} \\
\hline-24 & -37 & 4 & 1047 & Left Hippocampus (Hipp) & 30 & 5.23 \\
\hline-7 & -52 & 28 & & Left Posterior Cingulate Gyrus (PCG) & 31 & 3.63 \\
\hline-24 & -51 & 17 & & Left Precuneus & 30 & 3.55 \\
\hline-49 & 4 & -1 & 1566 & Left Superior Temporal Gyrus (STG) & 22 & 4.48 \\
\hline-42 & 29 & -2 & & Left Inferior Frontal Gyrus (IFG) & 47 & 3.86 \\
\hline-27 & 25 & -3 & & Left Insula & 13 & 3.61 \\
\hline \multicolumn{7}{|c|}{ Regional GMV increase in OSA follow $v$ s. OSA baseline } \\
\hline-15 & -81 & -27 & 1254 & Left Cerebellum & - & 4.18 \\
\hline
\end{tabular}

Gray matter volume (GMV) differences between groups are described in terms of MNI coordinates, voxel extent, brain side, and corresponding anatomical regions. The $\mathrm{t}$-score of the voxel with the strongest group effect in a given cluster is also listed. The statistical criteria of the VBM results were set as a cluster level FWE corrected $p<0.05$ (using a Monte Carlo simulation to correct the multiple comparison problem)

FWE family wised error; $L t$ left side; MNI Montreal Neurological Institute; Rt right side; voxel-based morphometry

ity and higher early leukocyte apoptosis. After treatment, recovery from increased GMV in pretreatment, particularly in the Hipp/PCG/Precuneus, highly correlated with improved leukocyte early apoptosis.

(4) OSA patients demonstrated persistently lower GMV in the anterior cingulate gyrus, even after surgical treatment.

Peripheral leukocyte apoptosis before and after treatment Changes in peripheral leukocyte apoptosis in OSA before and after surgery may partially explain the consequences and possible pathophysiology when patients experience repetitive episodes of hypoxia/re-oxygenation during the transient cessation of breathing [28]. Altered endothelial function is associated with enhanced release of specific leukocytes and systemic oxidative stress in OSA [29]. Previously, the use of CPAP has also been demonstrated to improve serum levels of C-reactive protein, tumor necrosis factor alpha, and interleukin-6, which can be further associated with extensive morbidity [30]. Increased early leukocyte apoptosis may be because some leukocyte injuries have not yet reached necrotic change [31] and provide the possibility of reducing activation of cell death receptors and mitochondria-dependent apoptotic pathways after aggressive treatment [32]. Like
CPAP, surgical treatment can also alleviate the increased systemic inflammation by decreasing early leukocyte apoptosis in patients with OSA.

\section{GMV alterations before and after surgery}

Although multiple pathophysiologies support the structural alterations occurring in OSA, differences in the studied patient population (sample size and disease severity), other co-morbidities, and different analysis procedures used with variable statistical criteria selection may lead to the differences observed between studies [8]. Structural changes in brain regions, including the frontal and parietal cortices, hippocampus, temporal gyrus, insula, cingulate gyri, caudate nucleus, and deep cerebellar nuclei, have been reported [9, 33, 34], and associated with different cognitive deficits [35]. According to previous reports [8], and this study, GMV reduction in the anterior cingulate gyrus before and after surgical treatment should be a hallmark of a patient with OSA. Physiologically, the cingulate gyrus, together with the insula, is mainly involved in cardiovascular control and may be damaged by intermittent hypoxia and sleep fragmentation.

Three months following surgery, a significant improvement in early leukocyte apoptosis was strengthened by the significant correlation with GMV reduction in the precuneus. However, the results here conflict with those 

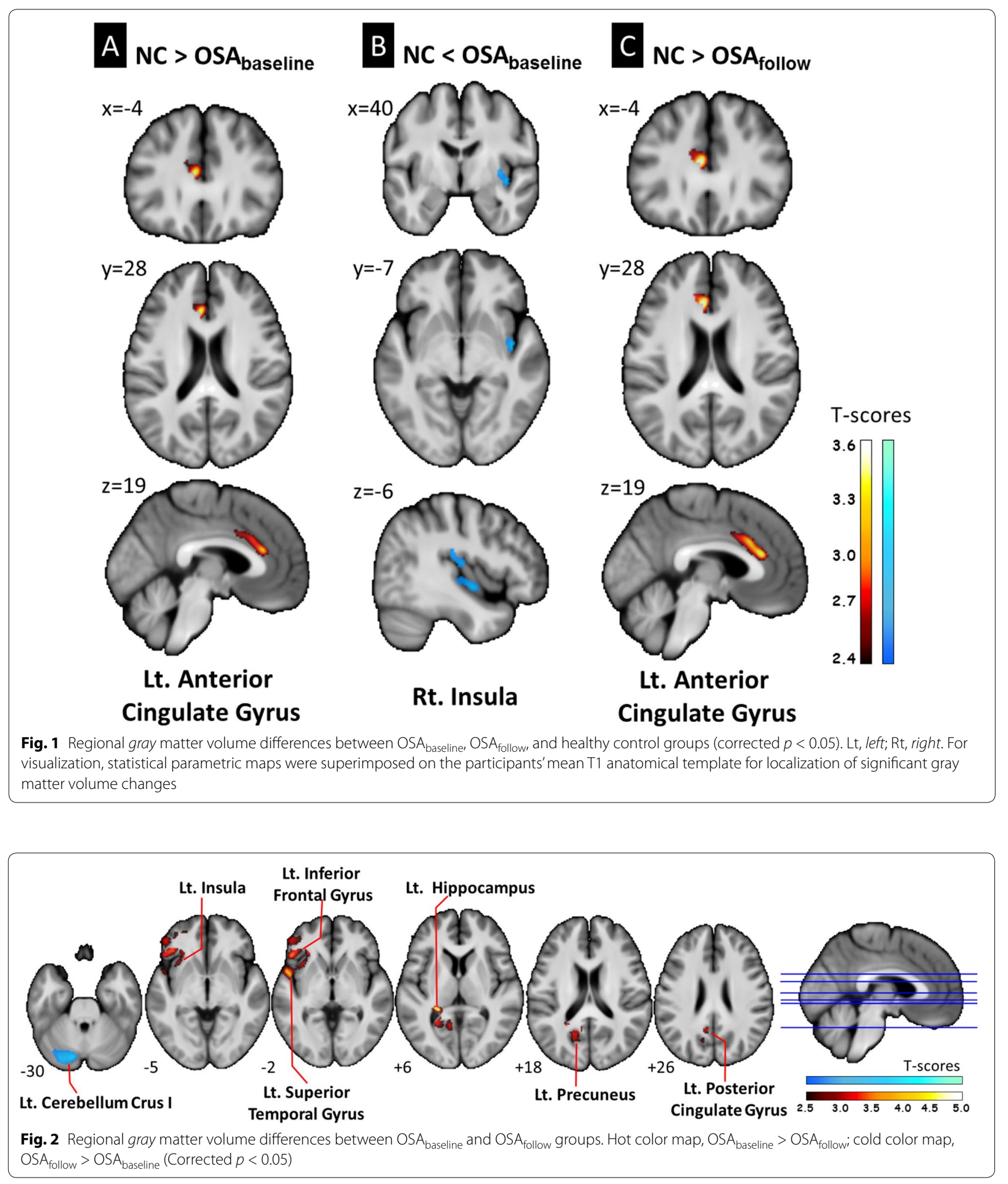

reported in other related VBM studies in patients with OSA. O'Donoghue et al. showed no significant structural difference in patients treated with CPAP between pre-treatment severe OSA (mean AHI, $71.7 \pm 7.0$ ) and control subjects, and no changes following therapy [36]. Huynh et al. also reported no differences in GMV between patients with moderate-to-severe OSA and healthy controls, and no difference between CPAP and 
Table 4 Correlation between regional GMV and clinical disease severity (PSG parameters and leukocyte apoptosis)

\begin{tabular}{|c|c|c|c|c|c|c|c|c|}
\hline \multicolumn{9}{|c|}{ Regional GMV differences between $\mathrm{OSA}_{\text {baseline }}$ and controls } \\
\hline & & \multicolumn{2}{|c|}{ AHI } & $\begin{array}{l}\text { Average } \mathrm{O}_{2} \\
\text { saturation }\end{array}$ & $\begin{array}{l}\text { De-saturation } \\
\text { index }\end{array}$ & $\begin{array}{l}\text { Granulocyte early } \\
\text { apoptosis }\end{array}$ & $\begin{array}{l}\text { Monocyte early } \\
\text { apoptosis }\end{array}$ & $\begin{array}{l}\text { Total leukocyte } \\
\text { early apoptosis }\end{array}$ \\
\hline \multirow{2}{*}{\multicolumn{2}{|c|}{$\begin{array}{l}\text { Left Anterior Cingulate } \\
\text { Gyrus }\end{array}$}} & r & -0.337 & 0.140 & -0.217 & -0.181 & -0.259 & -0.282 \\
\hline & & $p$ & 0.059 & 0.444 & 0.234 & 0.321 & 0.153 & 0.117 \\
\hline \multirow{2}{*}{\multicolumn{2}{|c|}{ Right Insula }} & r & \multirow{2}{*}{$\begin{array}{l}0.516 \\
0.003^{*}\end{array}$} & -0.574 & 0.508 & 0.419 & 0.364 & 0.405 \\
\hline & & $\mathrm{p}$ & & $0.001^{*}$ & $0.003^{*}$ & $0.017^{*}$ & $0.041^{*}$ & $0.022^{*}$ \\
\hline \multicolumn{9}{|c|}{ Regional GMV differences between OSA $A_{\text {baseline }}$ and OSA $A_{\text {follow }}$} \\
\hline & & \multicolumn{2}{|l|}{$\Delta \mathrm{AHI}$} & $\begin{array}{l}\Delta \text { Average } \mathrm{O}_{2} \\
\text { saturation }\end{array}$ & $\begin{array}{l}\Delta \text { De-saturation } \\
\text { index }\end{array}$ & $\begin{array}{l}\Delta \text { Granulocyte } \\
\text { early apoptosis }\end{array}$ & $\begin{array}{l}\Delta \text { Monocyte } \\
\text { early apoptosis }\end{array}$ & $\begin{array}{l}\Delta \text { Total leukocyte } \\
\text { early apoptosis }\end{array}$ \\
\hline \multirow{2}{*}{$\begin{array}{l}\text { Left Hipp/PCG/Pre- } \\
\text { cuneus }\end{array}$} & r & \multicolumn{2}{|l|}{-0.035} & -0.141 & 0.116 & 0.521 & 0.407 & 0.562 \\
\hline & $p$ & \multicolumn{2}{|l|}{0.885} & 0.565 & 0.637 & $0.022^{*}$ & 0.084 & $0.012^{*}$ \\
\hline \multirow[t]{2}{*}{ Left STG/IFG/Insula } & r & \multicolumn{2}{|l|}{0.231} & 0.106 & 0.096 & 0.058 & -0.199 & -0.075 \\
\hline & $p$ & \multicolumn{2}{|l|}{0.341} & 0.665 & 0.696 & 0.812 & 0.415 & 0.760 \\
\hline \multirow[t]{2}{*}{ Left cerebellum } & r & \multicolumn{2}{|l|}{0.201} & 0.311 & 0.167 & -0.376 & -0.438 & -0.442 \\
\hline & $p$ & \multicolumn{2}{|l|}{0.410} & 195 & 0.495 & 0.113 & 0.060 & 0.058 \\
\hline
\end{tabular}

Anatomical regions: Hipp/PCG/Precuneus, hippocampus/posterior cingulate gyrus/precuneus; STG/IFG/Insula, superior temporal gyrus/inferior frontal gyrus/insula AHI apnea-hypopnea index; GMV gray matter volume; OSA obstructive sleep apnea; PSG polysomnography

* Statistical threshold was set at $p<0.05$
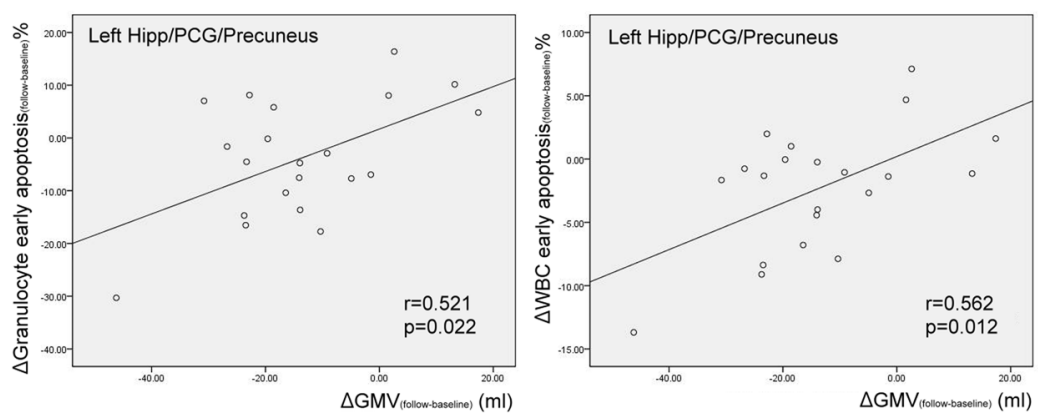

Fig. 3 Correlations between interval change of leukocyte profile and regional gray matter volume

sham CPAP treatment [10]. However, Canessa et al. [9] reported a positive effect of CPAP treatment for severe OSA (mean AHI, $55.8 \pm 19.1$ ). After treatment, improvements in memory, attention, and executive-functioning paralleled GMV increases in hippocampal and frontal structures.

The present study enrolled OSA patients ranging from mild to severe disease severity (AHI, $38.77 \pm 19.91$; range, 13-75) and found longitudinal changes in GMV after surgery. This is partially consistent with Canessa's observations. The difference in results may be explained by the different characteristics of the subject groups, different outcome measures and statistical criteria of imaging analyses (tissue volume vs. tissue concentration, uncorrected vs. corrected $p$-value), and different treatment effects between surgery and CPAP.

\section{Possible pathophysiology of GMV alterations}

The mechanism of increased GMV before treatment and reduction after treatment can be explained by short-term or reversible cerebral vasogenic edema, a phenomenon also found in other hypoxic conditions such as high altitude cerebral edema and acute mountain sickness [37, 38]. An increased permeability of the blood brain barrier (BBB) tight junctions with subsequent formation of mild extracellular brain edema has been shown as the "normal" sequence of events under hypoxia [39]. Pretreatment enlarged insular GMV positively correlating with increased hypoxic severity partially supports this hypothesis.

In the present study, elevated systemic leukocyte apoptosis before treatment in OSA may result from BBB breakdown, elevated peripheral mediators, and inflammation [40, 41]. In contrast, anti-inflammatory effects 
from interleukin, heat shock proteins, and adrenomedullin can improve BBB function [42]. We found that decreased granulocyte and total leukocyte early apoptosis were associated with GMV reduction in the Hipp/ PCG/Precuneus. Furthermore, correction of increased GMV by reducing hypoxic exposure with surgery may re-normalize cerebral autoregulation and reduce subsequent leukocyte reaction. The results here support the possibility that cytokine-mediated BBB damage in cardiovascular complications of OSA may partially contribute to alterations in GMV.

The present study has its limitations. Although there is improvement of cognitive functions after surgery, the report fails to demonstrate the relationship between them and systemic inflammation and GMV alteration, presumably because of the small sample size of OSA subjects. Furthermore, statistically significant associations, especially in small groups, do not necessarily mean a causal relationship. Partially corrected hypoxic status (AHI score, Pre-OP 38.77 vs. Post-OP 25.21) and nonrecovered brain injury (persistently decrease GMV in the anterior cingulate gyrus) after surgery may also explain the lesser improvement in neuropsychiatric functions. It is still too early to conclude on the long-term effects on brain structure, cognitive functions, and systemic inflammation from different treatments in OSA. Another limitation is that the healthy control subjects were not re-evaluated at 3 months, although changes in the GM of healthy participants over a short duration should be limited.

\section{Conclusions}

OSA can alter GM integrity in vulnerable regions, an effect associated with increased disease severity and systemic inflammation. The possible interactions between systemic inflammation and GM changes may represent variant hypoxic patterns and their consequent processes. Surgical treatment can partially reverse structural brain abnormalities with reduced systemic inflammation. This study may serve as a motivator for better treatment adherence by patients.

\section{Additional file}

Additional file 1. The procedure for the cross-sectional voxel-based morphometry pipeline.

\footnotetext{
Abbreviations

OSA: obstructive sleep apnea; GMV: gray matter volume; AHI: apnea-hypopnea index; CPAP: continuous positive airway pressure; VBM: voxel-based morphometry; GMV: gray matter volume; BMI: body mass index; MRI: magnetic resonance imaging; CASI: cognitive ability screening instrument; WAIS-III: Wechsler Adult Intelligence Scale; BDI: Beck Depression Inventory II; ANCOVA: analysis of covariance.
}

\section{Authors' contributions}

WCL participated in the project conception, organization, execution, statistical analysis, manuscript writing, review, and critique. CCH, PCC and MHC participated in the execution of statistical analysis. HLC participated in the project execution and statistical analysis review. KHC participated in the statistical analysis, including design, execution, manuscript writing, review, and critique. NWT participated in the project organization and execution. MF and $\mathrm{HCL}$ participated in the execution. CHL participated in the project conception, organization, execution, statistical analysis, manuscript writing, review, and critique. All authors read and approved the final manuscript.

\section{Author details}

${ }^{1}$ Department of Diagnostic Radiology, Kaohsiung Chang Gung Memorial Hospital, Chang Gung University College of Medicine, Kaohsiung, Taiwan. 2 Department of Neurology, Kaohsiung Chang Gung Memorial Hospital, Chang Gung University College of Medicine, Kaohsiung, Taiwan. ${ }^{3}$ Department of Biomedical Imaging and Radiological Sciences, National Yang-Ming University, Taipei, Taiwan. ${ }^{4}$ Department of Brain Research Center, National Yang-Ming University, Taipei, Taiwan. ${ }^{5}$ Division of Sleep Surgery, Department of Otolaryngology-Head and Neck Surgery, Rush University Medical Center, Chicago, IL, USA. ${ }^{6}$ Department of Otolaryngology, Advanced Center for Specialty Care, Advocate Illinois Masonic Medical Center, Chicago, IL, USA. ${ }^{7}$ Department of Otolaryngology, Kaohsiung Chang Gung Memorial Hospital, Chang Gung University College of Medicine, 123, Ta Pei Road, Niao Sung District, Kaohsiung, Taiwan. ${ }^{8}$ Sleep Center, Kaohsiung Chang Gung Memorial Hospital, Chang Gung University College of Medicine, Kaohsiung, Taiwan. ${ }^{9}$ Department of Biological Science, National Sun Yat-Sen University, Kaohsiung, Taiwan.

\section{Acknowledgements}

This work was supported by grants from the National Science Council of the Republic of China (NSC NMRPG 8B6142 to HL Chen, and NMRPG8E6051 and NMRPG8E6052 to CH Lu) and Chang Gung Medical Research Project (CMRPG $8 \mathrm{C} 0021$ to PC Chen). The authors wish to thank the MRI Core Facility of CGMH, as well as Yi-Wen Chen and Ting-Yi Chen, and all subjects who participated in this study.

\section{Competing interests}

The authors declare that they have no competing interests.

Received: 14 November 2015 Accepted: 28 April 2016 Published online: 17 May 2016

\section{References}

1. Lavie L, Polotsky V. Cardiovascular aspects in obstructive sleep apnea syndrome-molecular issues, hypoxia and cytokine profiles. Respiration. 2009;78:361-70.

2. Aloia MS, Arnedt JT, Davis JD, Riggs RL, Byrd D. Neuropsychological sequelae of obstructive sleep apnea-hypopnea syndrome: a critical review. J Int Neuropsychol Soc. 2004;10:772-85.

3. Lavie L, Dyugovskaya L, Polyakov A. Biology of peripheral blood cells in obstructive sleep apnea-the tip of the iceberg. Arch Physiol Biochem. 2008;114:244-54.

4. Carson MJ, Thrash JC, Walter B. The cellular response in neuroinflammation: the role of leukocytes, microglia and astrocytes in neuronal death and survival. Clin Neurosci Res. 2006;6:237-45.

5. Lee SJ, Benveniste EN. Adhesion molecule expression and regulation on cells of the central nervous system. J Neuroimmunol. 1999;98:77-88.

6. Kokturk O, Ciftci TU, Mollarecep E, Ciftci B. Elevated C-reactive protein levels and increased cardiovascular risk in patients with obstructive sleep apnea syndrome. Int Heart J. 2005;46:801-9.

7. Xu W, Chi L, Row BW, Xu R, Ke Y, Xu B, Luo C, Kheirandish L, Gozal D, Liu R. Increased oxidative stress is associated with chronic intermittent hypoxiamediated brain cortical neuronal cell apoptosis in a mouse model of sleep apnea. Neuroscience. 2004;126:313-23.

8. Weng HH, Tsai YH, Chen CF, Lin YC, Yang CT, Tsai YH, Yang CY. Mapping gray matter reductions in obstructive sleep apnea: an activation likelihood estimation meta-analysis. Sleep. 2014;37:167-75. 
9. Canessa N, Castronovo V, Cappa SF, Aloia MS, Marelli S, Falini A, Alemanno F, Ferini-Strambi L. Obstructive sleep apnea: brain structural changes and neurocognitive function before and after treatment. Am J Respir Crit Care Med. 2011;183:1419-26.

10. Huynh NT, Prilipko O, Kushida CA, Guilleminault C. Volumetric brain morphometry changes in patients with obstructive sleep apnea syndrome: effects of CPAP treatment and literature review. Front Neurol. 2014;5:58.

11. Kim H, Joo E, Suh S, Kim JH, Kim ST, Hong SB. Effects of long-term treatment on brain volume in patients with obstructive sleep apnea syndrome. Hum Brain Mapp. 2016;37:395-409.

12. Haniffa M, Lasserson TJ, Smith I: Interventions to improve compliance with continuous positive airway pressure for obstructive sleep apnoea. Cochrane Database Syst Rev 2004:CD003531.

13. Lin HS, Zuliani G, Amjad EH, Prasad AS, Badr MS, Pan CJ, Rowley JA. Treatment compliance in patients lost to follow-up after polysomnography. Otolaryngol Head Neck Surg. 2007;136:236-40.

14. Chen HL, Lu CH, Lin HC, Chen PC, Chou KH, Lin WM, Tsai NW, Su YJ, Friedman M, Lin CP, Lin WC. White matter damage and systemic inflammation in obstructive sleep apnea. Sleep. 2015;38:361-70.

15. Sleep-related breathing disorders in adults: recommendations for syndrome definition and measurement techniques in clinical research. The Report of an American Academy of Sleep Medicine Task Force. Sleep 1999, 22:667-689

16. Huang CC, Lin WC, Chen HL, Friedman M, Lin MC, Lin HC, Lu CH. Improvement of baroreflex sensitivity in patients with obstructive sleep apnea following surgical treatment. Clin Neurophysiol. 2016;127:544-50.

17. Lin HC, Friedman M, Chang HW, Gurpinar B. The efficacy of multilevel surgery of the upper airway in adults with obstructive sleep apnea/hypopnea syndrome. Laryngoscope. 2008;118:902-8.

18. Lin HC, Friedman M, Chang HW, Su MC, Wilson M. Z-palatopharyngoplasty plus radiofrequency tongue base reduction for moderate/ severe obstructive sleep apnea/hypopnea syndrome. Acta Otolaryngol. 2010;130:1070-6.

19. Lin HC, Friedman M, Chang HW, Yalamanchali S. Z-palatopharyngoplasty combined with endoscopic coblator open tongue base resection for severe obstructive sleep apnea/hypopnea syndrome. Otolaryngol Head Neck Surg. 2014;150:1078-85.

20. Lin WC, Tsai NW, Huang YC, Cheng KY, Chen HL, Li SH, Kung CT, Su YJ, Lin WM, Chen MH, et al. Peripheral leukocyte apoptosis in patients with Parkinsonism: correlation with clinical characteristics and neuroimaging findings. Biomed Res Int. 2014;2014:635923.

21. Lin WC, Chen PC, Wang HC, Tsai NW, Chou KH, Chen HL, Su YJ, Lin CP, Li $\mathrm{SH}$, Chang WN, Lu CH. Diffusion tensor imaging study of white matter damage in chronic meningitis. PLoS One. 2014;9:e98210.

22. Lin WC, Chou KH, Chen CL, Chen HL, Lu CH, Li SH, Huang CC, Lin CP, Cheng YF. Longitudinal brain white matter alterations in minimal hepatic encephalopathy before and after liver transplantation. PLoS One. 2014;9:e105887.

23. Teng EL, Hasegawa K, Homma A, Imai Y, Larson E, Graves A, Sugimoto K, Yamaguchi T, Sasaki H, Chiu D, et al. The Cognitive Abilities Screening Instrument (CASI): a practical test for cross-cultural epidemiological studies of dementia. Int Psychogeriatr. 1994;6(45-58):discussion 62.

24. Hsin-Yi Chen M-SH, Jianjun Zhu, Yung-Hwa Chen. Selection of FactorBased WAIS-III Tetrads in the Taiwan Standardization Sample: a guide to clinical practice. Chinese Journal of Psychology. 2008;50:91-109.

25. Beck AT, Steer RA, Ball R, Ranieri W. Comparison of beck depression inventories -IA and -II in psychiatric outpatients. J Pers Assess. 1996;67:588-97.

26. Chou KH, Lin WC, Lee PL, Tsai NW, Huang YC, Chen HL, Cheng KY, Chen PC, Wang HC, Lin TK, et al. Structural covariance networks of striatum subdivision in patients with Parkinson's disease. Hum Brain Mapp. 2015;36:1567-84.

27. Yang FC, Chou KH, Fuh JL, Huang CC, Lirng JF, Lin YY, Lin CP, Wang SJ. Altered gray matter volume in the frontal pain modulation network in patients with cluster headache. Pain. 2013;154:801-7.
28. Jelic S, Le Jemtel TH. Inflammation, oxidative stress, and the vascular endothelium in obstructive sleep apnea. Trends Cardiovasc Med. 2008;18:253-60.

29. Schulz R, Mahmoudi S, Hattar K, Sibelius U, Olschewski H, Mayer K, Seeger W, Grimminger F. Enhanced release of superoxide from polymorphonuclear neutrophils in obstructive sleep apnea. Impact of continuous positive airway pressure therapy. Am J Respir Crit Care Med. 2000;162:566-70.

30. Baessler A, Nadeem R, Harvey M, Madbouly E, Younus A, Sajid H, Naseem J, Asif A, Bawaadam H. Treatment for sleep apnea by continuous positive airway pressure improves levels of inflammatory markers - a meta-analysis. J Inflamm (Lond). 2013;10:13.

31. Kabara E, Coussens PM. Infection of primary bovine macrophages with mycobacterium avium subspecies paratuberculosis suppresses host cell apoptosis. Front Microbiol. 2012;3:215.

32. Dhar-Mascareno M, Carcamo JM, Golde DW. Hypoxia-reoxygenationinduced mitochondrial damage and apoptosis in human endothelial cells are inhibited by vitamin C. Free Radic Biol Med. 2005;38:1311-22.

33. Joo EY, Tae WS, Lee MJ, Kang JW, Park HS, Lee JY, Suh M, Hong SB. Reduced brain gray matter concentration in patients with obstructive sleep apnea syndrome. Sleep. 2010;33:235-41.

34. Macey PM, Henderson LA, Macey KE, Alger JR, Frysinger RC, Woo MA, Harper RK, Yan-Go FL, Harper RM. Brain morphology associated with obstructive sleep apnea. Am J Respir Crit Care Med. 2002;166:1382-7.

35. Morrell MJ, McRobbie DW, Quest RA, Cummin AR, Ghiassi R, Corfield DR. Changes in brain morphology associated with obstructive sleep apnea. Sleep Med. 2003;4:451-4.

36. O'Donoghue FJ, Briellmann RS, Rochford PD, Abbott DF, Pell GS, Chan CH, Tarquinio N, Jackson GD, Pierce RJ. Cerebral structural changes in severe obstructive sleep apnea. Am J Respir Crit Care Med. 2005;171:1185-90.

37. Mairer K, Gobel M, Defrancesco M, Wille M, Messner H, Loizides A, Schocke M, Burtscher M. MRI evidence: acute mountain sickness is not associated with cerebral edema formation during simulated high altitude. PLoS One. 2012;7:e50334.

38. Mairer K, Wille M, Burtscher M. The prevalence of and risk factors for acute mountain sickness in the Eastern and Western Alps. High Alt Med Biol. 2010;11:343-8

39. Bailey DM, Bartsch P, Knauth M, Baumgartner RW. Emerging concepts in acute mountain sickness and high-altitude cerebral edema: from the molecular to the morphological. Cell Mol Life Sci. 2009;66:3583-94.

40. Bailey DM, Evans KA, James PE, McEneny J, Young IS, Fall L, Gutowski M, Kewley E, McCord JM, Moller K, Ainslie PN. Altered free radical metabolism in acute mountain sickness: implications for dynamic cerebral autoregulation and blood-brain barrier function. J Physiol. 2009;587:73-85.

41. Subudhi AW, Panerai RB, Roach RC. Effects of hypobaric hypoxia on cerebral autoregulation. Stroke. 2010;41:641-6.

42. Julian CG, Subudhi AW, Wilson MJ, Dimmen AC, Pecha T, Roach RC. Acute mountain sickness, inflammation, and permeability: new insights from a blood biomarker study. J Appl Physiol. 1985;2011(111):392-9.

\section{Submit your next manuscript to BioMed Central and we will help you at every step:}

- We accept pre-submission inquiries

- Our selector tool helps you to find the most relevant journal

- We provide round the clock customer support

- Convenient online submission

- Thorough peer review

- Inclusion in PubMed and all major indexing services

- Maximum visibility for your research

Submit your manuscript at www.biomedcentral.com/submit 